
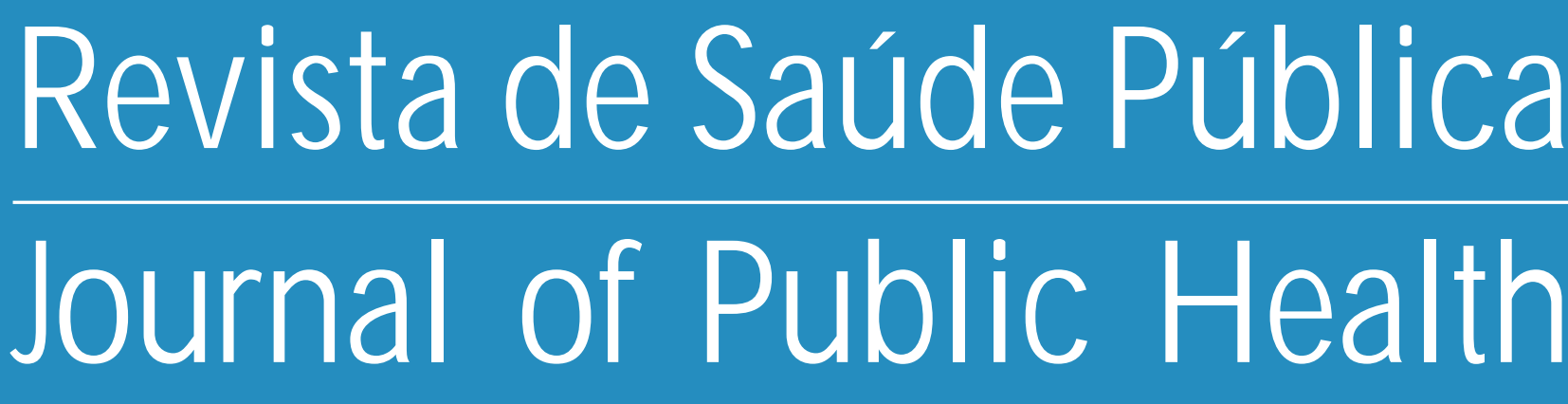

Cobertura vacinal e fatores de risco associados à não-vacinação em localidade urbana do Nordeste brasileiro, 1994*

Vaccination coverage and some risk factors

for non-vaccination in an urban area of N ortheastern Brazil, 1994

Antônio Augusto Moura da Silva, U ilho Antônio Gomes, Sueli Rosina Tonial e Raimundo Antonio da Silva

Departamento de Saúde Pública da Universidade Federal do Maranhão. São Luís, MA - Brasil (AAMS, SRT, RAS), Departamento de Medicina Social da Faculdade de Medicina de Ribeirão Preto da Universidade de São Paulo. Ribeirão Preto, SP - Brasil (UAG) 


\title{
Cobertura vacinal e fatores de risco associados à não-vacinação em localidade urbana do Nordeste brasileiro, 1994*
}

\section{Vaccination coverage and some risk factors for non-vaccination in an urban area of N ortheastern Brazil, 1994}

\author{
Antônio Augusto Moura da Silva, Uilho Antônio Gomes, Sueli Rosina Tonial e Raimundo \\ Antonio da Silva \\ Departamento de Saúde Pública da Universidade Federal do Maranhão. São Luís, MA - Brasil \\ (AAMS, SRT, RAS), Departamento de Medicina Social da Faculdade de Medicina de Ribeirão Preto \\ da Universidade de São Paulo. Ribeirão Preto, SP - Brasil (UAG)
}

\section{Descritores}

Programas de vacinação.

Extensão de cobertura.

\begin{abstract}
Resumo
Introdução

A identificação da cobertura vacinal e dos fatores responsáveis pelo retardo ou pela falta de imunizações é fundamental para a adequada monitorização dos programas de vacinação e para se identificar e atingir as crianças que não são vacinadas adequadamente.

\section{Métodos}

Foi realizado inquérito domiciliar transversal, em amostra aleatória por conglomerados em múltiplos estágios de crianças de 12 a 59 meses de idade, no Município de São Luís, Maranhão, Brasil, em 1994. Utilizou-se questionário padronizado respondido pela mãe ou responsável pela criança. Foram visitados 50 setores censitários; em cada um foram amostrados 40 domicílios, onde foram encontradas, em média, 15 crianças. $O$ efeito de desenho foi calculado para cada estimativa. A não-vacinação foi analisada em relação a indicadores socioeconômicos, demográficos e à morbidade referida pela regressão de Cox.
\end{abstract}

\section{Resultados}

A cobertura vacinal foi de $72,4 \%$ para BCG, $59,9 \%$ para 3 doses da vacina Sabin, $57 \%$ para 3 doses de vacina DPT (difteria, coqueluche e tétano) e 54,7\% para a vacina anti-sarampo. A baixa escolaridade materna foi o principal fator de risco para a não-vacinação após o controle dos fatores de confusão.

\section{Conclusão}

As coberturas vacinais foram baixas. Uma das estratégias sugeridas para o aumento das coberturas é o incremento das atividades de educação em saúde.
Correspondência para/Correspondence to: Antônio Augusto Moura da Silva Rua Barão de Itapary, 155

Centro - 65020-070 São Luís, MA - Brasil. E-mail: aasilva@elo.com.br
* Parte da tese de doutorado de A. A. M. da Silva, apresentada ao Departamento de Medicina Social da Faculdade de Medicina de Ribeirão Preto da USP, 1997.

Financiado pela Fundação de Amparo à Pesquisa do Estado do Maranhão/FAPEMA(Processo n ${ }^{\circ} 446 /$ 93), pelo Conselho Nacional de Desenvolvimento Científico e Tecnológico/CNPq, pela CAPES e pela Secretaria de Estado da Saúde e Secretaria Municipal de Saúde de São Luís.

Recebido em 26.11.1997.

Reapresentado em 23.7.1998.

Aprovado em 19.8.1998. 


\section{Keywords}

Immunization programs.

Extension of health services coverage.

\section{Abstract}

\section{Introduction}

The assessment of vaccination coverage and risk factors for non-vaccination is important to evaluate vaccination programs and to identify children not properly vaccinated.

\begin{abstract}
Methods
A cross-sectional household survey was carried out in the municipality of S. Luís, Maranhão, Brazil by means of a standardized questionnaire. Multistage cluster sampling was used to identify children of 12-59 months of age residing in the city in 1994. The mother or other person responsible for the children was interviewed. Fifty census clusters were visited and 40 households were sampled in each. On average, 15 children were found in each cluster. Design effect was calculated for each estimate. Health service utilization was analyzed according to socioeconomic and demographic indicators, and perceived morbidity using proportional hazard modeling (Cox's regression).

Results

Vaccination coverage levels were $72.4 \%$ for BCG, 59.9\% for three doses of polio vaccine, 57\% for three doses of DTP vaccine and $54.7 \%$ for measles vaccine. Vaccination levels have remained statistically unchanged over the last three years. Lower maternal schooling continues to be associated with increased risk of nonvaccination in the multivariable analysis.
\end{abstract}

\section{Conclusion}

Vaccination levels were low. Health education activities are one of the suggested strategies to increase vaccination coverage.

\section{INTRO DUÇÃO}

A vacinação das crianças no primeiro ano de vida é fundamental para a prevenção de várias doenças transmissíveis e é um dos fatores associados com a redução da taxa de mortalidade infantil ${ }^{10,16}$. A identificação da cobertura vacinal e dos fatores responsáveis pelo retardo ou pela falta de imunizações é fundamental para a adequada monitorização dos programas de vacinação e para se identificar e atingir as crianças que não são vacinadas ${ }^{22,35}$.

No Brasil, especialmente a partir de 1973, quando o Ministério da Saúde criou o PNI (Programa Nacional de Imunizações), têm sido realizadas pesquisas de avaliação da cobertura vacinal no intuito de monitorar o cumprimento da meta de vacinar $100 \%$ das crianças menores de 1 ano com todas as vacinas do esquema básico ${ }^{35}$. No início da década de 80 , as coberturas vacinais no Brasil estavam bem abaixo da meta preconizada. Na PNAD (Pesquisa Nacional por Amostra de Domicílios) de 1981, dentre as crianças de 1 a 4 anos, 74,1\% estavam vacinadas contra o sarampo, 65,9\% tinham recebido a BCG, $74,9 \%$ receberam 3 doses da vacina DPT (difteria, coqueluche e tétano) e 94,4\% haviam tomado 3 doses da vacina Sabin. Apenas $38,3 \%$ das crianças ti- nham recebido todas estas citadas vacinas e completado o esquema básico de vacinação ${ }^{3}$. Em 1996, na Pesquisa Nacional sobre Demografia e Saúde ${ }^{4}$ mostrou-se que as coberturas vacinais aumentaram, comparando-se com a década anterior, atingindo $92,6 \%$ para BCG, $80,3 \%$ para 3 doses da vacina DPT, 80,7\% para 3 doses da vacina Sabin, $87,2 \%$ para a vacina anti-sarampo; $72,5 \%$ tomaram todas as vacinas.

Além de não ter sido atingida a meta do Ministério da Saúde, as desigualdades regionais nas coberturas vacinais são grandes, pois as mesmas são mais elevadas nos Estados do Sul e Sudeste ${ }^{7,9,12}$ e mais baixas na região Nordeste ${ }^{39,40,41}$. Em alguns Estados do Nordeste, como no Maranhão, em 1991, apenas $21,7 \%$ das crianças de 12 a 23 meses no Maranhão e $58,3 \%$ em São Luís tinham completado o esquema básico de vacinação ${ }^{17}$.

Apesar da melhoria nas coberturas vacinais observada no Brasil, uma parcela das crianças continua sem ser vacinada adequadamente, mesmo em locais com ampla disponibilidade de serviços de saúde. Vários estudos de avaliação da cobertura vacinal e dos fatores relacionados à não-vacinação realizados em amostras representativas da população infantil foram desenvolvidos no mundo e no Brasil no sentido de elucidar esta questão. Dentre os fatores 
de risco para a não-vacinação destacam-se: baixa renda $^{3,23,37}$, residência em área rural ${ }^{4,25,39,41}$, extremos de idade materna ${ }^{25}$, maior número de filhos ${ }^{4,14,25,40}$, baixa escolaridade materna $a^{4,14,37,40,41}$, maior número de moradores no domicílio ${ }^{40}$, residência há menos de 1 ano na área ${ }^{14}$, falta de conhecimento acerca das doenças preveníveis por imunização ${ }^{37,40}$, dificuldades de transporte, conflitos trabalhistas motivados pela perda de dias de trabalho para o cuidado dos filhos ${ }^{29}$, ausência de seguro-saúde ${ }^{23,24}$ e presença de doença na criançą ${ }^{40}$.

Não somente fatores relacionados com os usuários estão associados a níveis mais baixos de cobertura vacinal. Fatores estruturais relacionados aos serviços de saúde tais como retardo no agendamento das consultas, falta de consultas noturnas ou nos finais de semana, filas, tempo de espera, falta de brinquedos e distrações para as crianças durante a espera também dificultam as vacinações ${ }^{29}$. Outra causa da não-vacinação ligada aos serviços de saúde são as oportunidades vacinais perdidas. Estas ocorrem quando a criança não é vacinada na presença de doença leve ou quando a criança comparece à consulta na unidade de saúde na época de receber a vacina e não é vacinada ${ }^{14,44,45}$.

O objetivo do presente estudo é identificar alguns motivos da persistência de baixos níveis de coberturas vacinais no Município de São Luís, Maranhão, e contribuir para a identificação de clientelas não atingidas pela vacinação e para a elaboração de estratégias que possam elevar estas coberturas.

\section{MÉTO DOS}

O Município de São Luís localiza-se na ilha do Maranhão, ao norte do Estado do Maranhão, tendo uma área de $518 \mathrm{~km}^{2}$. Em 1996, sua população era de 781.068 habitantes ${ }^{19}$. Teve forte crescimento demográfico ao longo das últimas décadas, apesar da taxa de crescimento populacional estar diminuindo. A estrutura dos serviços de saúde, especialmente a rede de ambulatórios públicos, aumentou bastante nos últimos anos ${ }^{20}$.

Foi realizado estudo transversal, utilizando o método de amostragem por conglomerados em três estágios. No cálculo do tamanho amostral utilizou-se a fórmula $\mathrm{n}=\left(\mathrm{z}^{2} \times \mathrm{p} \times \mathrm{q}\right) / \mathrm{d}^{2}$, supondo-se amostragem casual simples. Fixou-se a probabilidade de erro tipo I em $5 \%$ e a precisão absoluta desejada em torno da estimativa em 5\%. Como se estimaram muitas variáveis na mesma amostra, calculou-se o produto máximo de p x q (50\%), no sentido de obter-se amostra de maior abrangência possível. A amostra final necessária foi em torno de 400 crianças. Como a amostragem não foi casual simples, estimou-se efeito de desenho em torno de 2 para os indicadores, necessitandose em torno de 800 crianças na amostra, considerando-se a amostragem por conglomerados ${ }^{26}$.

No primeiro estágio foram sorteados, de forma sistemática, 50 dos 578 setores censitários do IBGE, a partir de listagem ordenada dos mesmos, com probabilidade proporcional ao número de domicílios particulares ocupados (base censitária de 1991) ${ }^{18}$. No segundo estágio foram sorteados os quarteirões, e no terceiro foram sorteados os domicílios para serem visitados após a realização de censo no quarteirão sorteado, ambos por amostragem aleatória simples. Em cada setor foram visitados 40 domicílios, com média de 15 crianças por setor. $\mathrm{O}$ percentual de recusas e ausências foi de $6,8 \%$, após 3 repasses em dias e horários diferentes, semelhante ao de outros trabalhos ${ }^{17,41}$. Não se fez substituição de domicílios nos casos de perda.

Foi utilizado questionário padronizado para coleta de dados, respondido pela mãe ou responsável. O questionário foi bastante similar ao utilizado na PESN (Pesquisa Estadual de Saúde e Nutrição - Maranhão) e em várias pesquisas realizadas nos Estados do Nordeste $^{2}$.

As variáveis estudadas foram: vacinação, gênero e idade da criança, escolaridade paterna e materna (em anos completos de freqüência à escola), renda familiar em salários-mínimos, ocupação do chefe de família (não manual, manual especializada ou semi-especializada e manual não qualificada ou desempregado - adaptada da Classificação Internacional Padrão de Ocupações - ISCO-68) ${ }^{36}$, idade materna, número de irmãos residindo no domicílio, número de moradores residindo na casa, posse de segurosaúde (incluindo-se qualquer modalidade de assistência médica supletiva do tipo pré-pagamento) e morbidade referida nos três últimos meses.

Em relação à vacinação, quando a criança possuía o cartão de vacinas ou de saúde, anotavam-se do cartão as datas em que recebeu a terceira dose das vacinas DPT e Sabin, BCG e anti-sarampo. Se a criança não tinha o cartão, perguntava-se sobre o número de doses recebidas das vacinas BCG, Sabin, anti-sarampo e DPT para a mãe ou responsável. Somente considerava-se a resposta se esta descrevesse, precisamente, a técnica de vacinação. A partir destas perguntas, calculou-se a cobertura vacinal em crianças de 12 a 59 meses segundo três critérios: confirmada pelo cartão de vacinas, considerando-se também as informações verbais prestadas pela mãe ou responsável, e cobertura vacinal na idade aconselhada (se tomou as vacinas no mês recomendado). Para fins de comparação com a PESN ${ }^{17}$, calculou-se também a cobertura vacinal, com base no cartão, em crianças de 12 a 23 meses. Considerou-se completado o esquema básico de vacinação quando a criança já havia recebido 3 doses de Sabin e DPT e uma dose de BCG e anti-sarampo antes de completar um ano de vida. 
A coleta de dados foi realizada de 15 de agosto a 25 de setembro de 1994, por estudantes universitários ou recém-graduados em medicina ou enfermagem. Os entrevistadores receberam treinamento para aplicação das entrevistas e participaram de estudo-piloto. O trabalho de campo foi realizado em duplas, tendo um supervisor de campo para cada dois entrevistadores. Os supervisores foram docentes de ensino superior da área da saúde.

A análise estatística incluiu cálculo da cobertura vacinal e da razão de prevalências (risco relativo). O risco foi sempre calculado em relação à categoria basal, considerada aquela com menor risco para a não-vacinação. Na comparação entre as estimativas obtidas na PESN, em $1991^{17}$, e as estimativas obtidas no presente trabalho, foi utilizado o teste "t" de Student para comparação entre duas proporções (amostras independentes).

As estimativas pontuais e por intervalo de confiança de $95 \%$ das proporções foram calculadas no programa CSAMPLE, do Epi Info, versão $6.04^{15}$, sendo o setor censitário a unidade primária de amostragem. O número de crianças estudadas por setor variou de 2 a 33 , sendo em média de 15 . Foram utilizadas estimativas não ponderadas no presente trabalho, pois a diferença observada entre as estimativas ponderadas e não ponderadas foi geralmente inferior a $1 \%$.

O efeito de desenho ${ }^{27}$ foi calculado para cada estimativa. Quando se utiliza amostragem por conglomerados, a variância das estimativas tende a ser maior em comparação à amostragem aleatória simples. Os intervalos de confiança são mais largos e a precisão das estimativas é, portanto, menor ${ }^{31}$. Os cálculos do intervalo de confiança e do efeito de desenho levaram em conta estas diferenças no tamanho das unidades primárias de amostragem. Os cálculos assumiram amostragem com reposição, pois a fração amostral foi baixa $(0,9 \%)$, sendo estudadas 748 das 82.403 crianças menores de 5 anos de São Luís ${ }^{18}$. No presente trabalho foram utilizados os dados das crianças de 12 a 59 meses, pois a cobertura vacinal é convencionalmente calculada excluindo-se os menores de 1 ano $^{3,4,17,41}$, pois estas crianças ainda têm oportunidade de serem vacinadas antes de completar o primeiro aniversário.

No ajuste para fatores de confusão, empregou-se a regressão de Cox $^{13}$ modificada por Breslow ${ }^{6}$. Breslow modificou a regressão de Cox impondo uma condição de duração constante de seguimento $(\mathrm{t}=1)$ para todos os indivíduos, tornando este método típico dos estudos de coorte aplicável a estudos transversais. Este modelo produz estimativa direta da razão de prevalências ajustada e respectivo intervalo de confiança de $95 \%$.

Foram utilizados dois processos de modelagem: o modelo completo e o modelo reduzido ${ }^{28}$. No completo todas as variáveis acima indicadas foram incluídas. A razão de prevalências obtida a partir do modelo completo foi uma estimativa ajustada para todas as outras variáveis analisadas. O modelo reduzido foi obtido por meio do módulo em passos, "stepwise", utilizando-se o processo de seleção para frente, "forward". Todas as variáveis associadas com a não-vacinação a um nível de significância de 0,20 na análise univariável foram incluídas no modelo e ficaram apenas aquelas que permaneceram associadas a um nível de significância de pelo menos 0,10. A significância de cada variável no modelo foi verificada pelo teste da razão de verossimilhanças. Todas as variáveis foram incluídas na análise como categóricas.

\section{RESU LTADOS}

\section{Cobertura Vacinal Confirmada pelo Cartão de Vacinas}

A vacinação foi medida pela cobertura vacinal para BCG, 3 doses de Sabin, 3 doses de DPT, uma dose de anti-sarampo e pelo esquema básico de vacinação completo no primeiro ano de vida. Avaliaram-se, inicialmente, apenas as informações obtidas a partir do cartão de vacinas e estimou-se a cobertura vacinal em 596 crianças de 12 a 59 meses, excluindo-se os casos com informação ilegível ou registrada de forma incorreta no cartão de vacinas. Dentre estas, $488(81,9 \%)$ possuíam o cartão de vacinas e este foi visto pelo entrevistador. Observouse cobertura de $72,4 \%$ para BCG, $59,9 \%$ para 3 doses de Sabin, 57,0\% para 3 doses de DPT e 54,7\% para uma dose de anti-sarampo. Apenas $44,1 \%$ das crianças estavam completamente vacinadas (Tabela 1).

A cobertura vacinal em crianças de 12 a 23 meses foi maior do que aquela calculada a partir daquelas com 12 a 59 meses. Observou-se cobertura de $80,3 \%$ para BCG, 68,8\% para 3 doses de Sabin, 64,1\% para 3 doses de DPT e 60,5\% para anti-sarampo. Apenas $52,7 \%$ das crianças estavam completamente vacina-

Tabela 1 - Cobertura vacinal confirmada pelo cartão de vacinas em crianças de 12 a 59 meses de idade, São Luís, 1994.

\begin{tabular}{lccccc}
\hline Vacinas & $\mathrm{n}$ & Total * & $\%$ & IC 95\% & Efeito de desenho \\
\hline BCG & 415 & 573 & 72,4 & $67,8-77,0$ & 1,585 \\
Sabin - 3 doses & 351 & 586 & 59,9 & $55,6-64,2$ & 1,160 \\
DPT - 3 doses & 334 & 586 & 57,0 & $53,1-60,9$ & 0,967 \\
Anti-sarampo & 317 & 580 & 54,7 & $49,9-59,4$ & 1,381 \\
Esquema vacinal básico & 246 & 558 & 44,1 & $39,2-48,9$ & 1,388 \\
\hline
\end{tabular}

IC - Intervalo de Confiança

* O s totais diferem para cada vacina devido a informações ilegíveis ou registradas de forma incorreta no cartão de vacinas. 
Tabela 2 - Cobertura vacinal confirmada pelo cartão de vacinas em crianças de 12 a 23 meses de idade, São Luís, 1994.

\begin{tabular}{lccccc}
\hline Vacinas & $\mathrm{n}$ & Total* & $\%$ & IC 95\% & Efeito de desenho \\
\hline BCG & 122 & 152 & 80,3 & $73,8-86,8$ & 1,060 \\
Sabin - 3 doses & 108 & 157 & 68,8 & $60,8-76,8$ & 1,209 \\
DPT - 3 doses & 100 & 156 & 64,1 & $56,1-72,1$ & 1,116 \\
Anti-sarampo & 92 & 152 & 60,5 & $51,9-69,1$ & 1,219 \\
Esquema vacinal básico & 77 & 146 & 52,7 & $44,5-61,0$ & 1,045 \\
\hline
\end{tabular}

IC - Intervalo de Confiança

* O s totais diferem para cada vacina devido a informações ilegíveis ou registradas de forma incorreta no cartão de vacinas.

das (Tabela 2). Comparando-se os resultados com os dados da PESN, 1991, observou-se que não houve alteração nos níveis de cobertura vacinal entre 1991 e 1994, em São Luís (Tabela 3).

\section{Cobertura Vacinal Considerando-se Também as Informações Verbais}

Analisou-se, também, a cobertura vacinal considerando-se os dados anotados no cartão de vacinas e também a informação sobre a vacinação fornecida pela mãe ou responsável. As coberturas foram mais elevadas. $\mathrm{O}$ percentual de crianças que havia completado o esquema básico de vacinação foi maior em 17\% (Tabela 4).

Tabela 3 - Cobertura vacinal confirmada pelo cartão de vacinas em crianças de 12 a 23 meses de idade, São Luís, 1991 e 1994.

\begin{tabular}{lccc}
\hline Vacinas & $\begin{array}{c}1991^{*} \\
\%\end{array}$ & $\begin{array}{c}1994 \\
\%\end{array}$ & $p$ \\
\hline BCG & 84,6 & 80,3 & 0,267 \\
Sabin - 3 doses & 60,0 & 68,8 & 0,097 \\
DPT - 3 doses & 57,6 & 64,1 & 0,217 \\
Anti-sarampo & 66,6 & 60,5 & 0,253 \\
\hline
\end{tabular}

Fonte: Pesquisa Estadual de Saúde e Nutrição (PESN $)^{17}, 1992$.

\section{Cobertura Vacinal na Idade Aconselhada}

Utilizou-se como indicador de qualidade dos serviços de vacinação a cobertura vacinal na idade aconselhada, ou seja, considerou-se vacinada adequadamente apenas a criança que recebeu BCG no primeiro mês, a terceira dose de Sabin e DPT com até 6 meses e anti-sarampo até os 9 meses de vida. Os níveis de cobertura vacinal na idade aconselhada foram muito baixos (Tabela 5).

\section{Análise não Ajustada dos Fatores Associados à Não-Vacinação}

Não foi observada associação entre vacinação e gênero da criança. As crianças de 1 e 2 anos foram vacinadas em maior proporção $(50,7 \%)$ do que as de 3 e 4 anos $(36,6 \%)$ (Tabela 6).

A cobertura vacinal foi menor para as crianças de mães que freqüentaram até 4 anos de escola $(30,6 \%)$ do que para aquelas cujas mães freqüentaram 9 anos de escola ou mais $(51,5 \%)$. Não houve diferença estatisticamente significante na cobertura

Tabela 4 - Cobertura vacinal baseada no cartão de vacinas e nas informações da mãe em crianças de 12 a 59 meses de idade, São Luís, 1994.

\begin{tabular}{lccccc}
\hline Vacinas & $\mathrm{n}$ & Total* & $\%$ & IC 95\% & Efeito de desenho \\
\hline BCG & 522 & 573 & 91,1 & $87,8-94,4$ & 1,998 \\
Sabin - 3 doses & 443 & 586 & 75,6 & $71,2-80,0$ & 1,578 \\
DPT - 3 doses & 414 & 586 & 70,6 & $66,1-75,2$ & 1,555 \\
Anti-sarampo & 404 & 580 & 69,7 & $64,9-74,4$ & 1,585 \\
\hline
\end{tabular}

IC - Intervalo de Confiança

* O s totais diferem para cada vacina devido a informações ilegíveis, registradas de forma incorreta no cartão de vacinas ou porque a mãe e/ou responsável não soube informar do estado vacinal da criança.

Tabela 5 - Cobertura vacinal na idade recomendada baseada no cartão de vacinas em crianças de 12 a 59 meses de idade, São Luís, 1994.

\begin{tabular}{lccccc}
\hline Vacinas & $\mathrm{n}$ & Total* & $\%$ & IC 95\% & Efeito de desenho \\
\hline BCG & 314 & 573 & 54,8 & $50,1-59,5$ & 1,343 \\
Sabin - 3 doses & 175 & 586 & 29,9 & $24,8-34,9$ & 1,880 \\
DPT - 3 doses & 157 & 586 & 26,8 & $22,1-31,5$ & 1,711 \\
Anti-sarampo & 214 & 580 & 36,9 & $32,4-41,4$ & 1,311 \\
\hline
\end{tabular}

IC - Intervalo de Confiança

* Os totais diferem para cada vacina devido a informações ilegíveis ou registradas de forma incorreta no cartão de vacinas. 
Tabela 6 - Esquema básico de vacinação em crianças de 12 a 59 meses de idade segundo gênero, idade da criança e alguns indicadores socioeconômicos, São Luís, 1994.

\begin{tabular}{|c|c|c|c|c|}
\hline Variáveis & Completa (\%) & $n *(596)$ & RP Bruta & RP Ajustada** \\
\hline \multicolumn{5}{|l|}{ Gênero } \\
\hline M asculino & 42,6 & 258 & & \\
\hline $\begin{array}{c}\text { Feminino } \\
\text { Idade da criança }\end{array}$ & 45,3 & 300 & $0,95(0,82-1,10)$ & $1,09(0,80-1,47)$ \\
\hline 1 e 2 anos & 50,7 & 296 & & \\
\hline 3 e 4 anos & 36,6 & 262 & $1,28(1,11-1,49) * * *$ & $1,18(0,87-1,60)$ \\
\hline \multicolumn{5}{|l|}{ Escolaridade paterna } \\
\hline 9 anos e mais & 45,8 & 214 & & \\
\hline 5 a 8 anos & 48,2 & 168 & $0,96(0,79-1,16)$ & $0,83(0,56-1,24)$ \\
\hline Até 4 anos & 38,9 & 131 & $1,13(0,94-1,35)$ & $0,88(0,58-1,34)$ \\
\hline \multicolumn{5}{|l|}{ Escolaridade materna } \\
\hline 9 anos e mais & 51,5 & 227 & & \\
\hline 5 a 8 anos & 45,4 & 194 & $1,13(0,94-1,36)$ & $1,23(0,81-1,87)$ \\
\hline Até 4 anos & 30,6 & 121 & $1,43(1,20-1,71)^{* * *}$ & $1,67(1,05-2,64) * * *$ \\
\hline \multicolumn{5}{|l|}{ Renda familiar } \\
\hline + 3 salários-mínimos & 48,0 & 177 & & \\
\hline +1 a 3 salários-mínimos & 45,4 & 218 & $1,05(0,87-1,27)$ & $0,86(0,57-1,30)$ \\
\hline Até 1 salário-mínimo & 38,5 & 135 & $1,18(0,97-1,44)$ & $0,99(0,62-1,59)$ \\
\hline \multicolumn{5}{|l|}{ O cupação do chefe de família } \\
\hline Não manual & 46,9 & 113 & & \\
\hline M anual especializada ou & & & & \\
\hline semi-especializada & 49,8 & 235 & $0,95(0,76-1,17)$ & $1,19(0,73-1,94)$ \\
\hline $\begin{array}{l}\text { Não qualificada/ } \\
\text { desempregado }\end{array}$ & 36,3 & 204 & $1,20(0,98-1,47)$ & $1,26(0,74-2,15)$ \\
\hline
\end{tabular}

* As informações ignoradas ou não registradas não foram incluídas nos cálculos

** Razão de prevalências ajustada segundo modelo completo de regressão

**** Variáveis significantes de $(p<0,05)$

vacinal segundo escolaridade paterna, renda familiar e ocupação do chefe de família (Tabela 6).

Não foi observada associação entre vacinação com idade materna, número de moradores na casa e morbidade referida. Houve diferença na vacinação segundo o número de irmãos no domicílio. Aqueles com até 2 irmãos completaram o esquema básico de vacinação em 46,9\% das vezes, ao passo que dentre aqueles com mais de 2 irmãos, só 30,3\% tinham a vacinação completa. Não houve diferença na cobertura vacinal comparando-se usuários e nãousuários de seguro-saúde (Tabela 7).

Tabela 7 - Esquema vacinal básico em crianças de 12 a 59 meses de idade segundo alguns indicadores demográficos, posse de seguro-saúde e morbidade referida, São Luís, 1994.

\begin{tabular}{|c|c|c|c|c|}
\hline Variáveis & Completa (\%) & $n *(596)$ & RP Bruta & RP Ajustada** \\
\hline \multicolumn{5}{|l|}{ Idade materna } \\
\hline 20 anos e mais & 45,2 & 502 & & \\
\hline Menor de 20 anos & 44,4 & 36 & $1,01(0,75-1,37)$ & $1,40(0,80-2,43)$ \\
\hline \multicolumn{5}{|l|}{ Número de irmãos } \\
\hline 0 a 2 & 46,9 & 467 & & \\
\hline 3 a 9 & 30,3 & 89 & $1,31(1,12-1,54)^{* * *}$ & $1,25(0,80-1,97)$ \\
\hline \multicolumn{5}{|l|}{ Moradores na casa } \\
\hline 1 a 5 & 48,2 & 299 & & \\
\hline 6 a 18 & 39,7 & 257 & $1,16(1,00-1,35)$ & $0,95(0,67-1,34)$ \\
\hline \multicolumn{5}{|l|}{ Seguro-saúde } \\
\hline Sim & 51,3 & 119 & & \\
\hline N ão & 42,3 & 437 & $1,18(0,97-1,45)$ & $1,28(0,79-2,08)$ \\
\hline \multicolumn{5}{|l|}{ Morbidade referida } \\
\hline Não & 44,2 & 199 & & \\
\hline Sim & 44,3 & 357 & $1,00(0,86-1,17)$ & $1,07(0,78-1,46)$ \\
\hline
\end{tabular}

* As informações ignoradas ou não registradas não foram incluídas nos cálculos 


\section{Análise Ajustada dos Fatores Associados à Não-Vacinação}

Após o ajuste para fatores de confusão, no modelo reduzido, as variáveis que explicaram a vacinação básica completa foram idade da criança e escolaridade materna. No modelo completo, somente as crianças de mães com até 4 anos de escola tiveram maior risco de não serem vacinadas. $\mathrm{O}$ maior número de irmãos, que está associado ao maior risco de não receber vacinação básica completa na análise não ajustada, não continuou associado após o controle dos fatores de confusão. Crianças com 3 e 4 anos tiveram um risco 1,29 vezes maior de não serem vacinadas comparadas às crianças mais jovens. O risco de uma criança de mãe que freqüentou até 4 anos de escola não ser vacinada foi 1,42 vezes maior em relação às crianças de mães de maior escolaridade (Tabelas 6 a 8).

Tabela 8 - Estimativas da razão de prevalências ajustada obtidas a partir do modelo reduzido da regressão de Cox para não-vacinação de crianças de 12 a 59 meses de idade, São Luís, 1994.

\begin{tabular}{ll}
\hline Variáveis & $\begin{array}{c}\text { Razão de } \\
\text { prevalências } \\
\text { ajustada }\end{array}$ \\
\hline Idade de 3 e 4 anos & $1,29(1,03-1,61)^{*}$ \\
Mãe com até 4 anos de escolaridade & $1,12(0,86-1,46)$ \\
Mãe com 5 a 8 anos de escolaridade & $1,42(1,07-1,88)^{*}$ \\
\hline * Variáveis significantes de $(p<0,05)$ &
\end{tabular}

\section{DISCUSSÃO E CONCLUSÕ ES}

Como o desenho do estudo foi adequado e o índice de perdas foi de $6,8 \%$ pode-se considerar esta amostra como representativa da população de crianças menores de 5 anos, residentes no Município de São Luís. A composição da população segundo renda familiar e escolaridade materna foi semelhante à composição da população de famílias com crianças menores de cinco anos observada no censo de $1991^{42}$, o que torna a ocorrência de vício de seleção improvável.

É possível que as coberturas vacinais estejam superestimadas em até $5 \%$, pois só foram estudadas crianças sobreviventes e estas tendem a ter coberturas mais altas do que as falecidas (estimando-se o coeficiente de mortalidade em menores de 5 anos em 50 por mil). As informações relativas à cobertura vacinal são confiáveis, pois considerou-se as vacinas anotadas no cartão e aquelas referidas pela mãe apenas quando esta descreveu adequadamente a técnica de vacinação empregada.

Uma das limitações do presente estudo é que as famílias com mais de uma criança menor de 5 anos estão representadas mais de uma vez (o que ocorreu em $22,1 \%$ dos casos), o que pode ter contribuído para diminuir a precisão das estimativas e aumentar o efeito de desenho. Entretanto, o maior efeito de desenho observado foi 2 , indicando que a correção utilizada no cálculo do tamanho amostral foi adequada.

As taxas de não-resposta a quesitos específicos foram maiores para escolaridade paterna $(13,9 \%) \mathrm{e}$ renda familiar $(11,1 \%)$. Em relação às coberturas vacinais, a taxa de não resposta foi mais baixa para as vacinas DPT e Sabin $(1,7 \%)$ e mais alta para o esquema vacinal básico completo $(6,4 \%)$. Trabalho realizado em Ribeirão Preto apresentou taxas de não resposta mais altas, de 29,2\% para a renda familiar e de $16,2 \%$ para a escolaridade paterna ${ }^{5}$. Em Pelotas, a taxa de não-resposta para a renda familiar foi mais baixa, de $9,8 \%{ }^{33}$.

A razão de prevalências utilizada como medida de risco é mais adequada para estudos transversais do que a razão de chances (odds ratio). Como a nãovacinação para todas as vacinas do esquema básico no primeiro ano de vida (variável resposta) foi de $55,9 \%$, o pressuposto de raridade não é verdadeiro e, deste modo, a razão de chances estimada pelo modelo logístico é um indicador viciado. Utilizando-se a razão de chances há uma tendência à superestimação dos riscos, aumentando a probabilidade de erro do tipo I, e à diminuição da precisão das estimativas, pois os intervalos de confiança da razão de chances tendem a ser mais alargados quando o evento é freqüente ${ }^{6,13,30}$.

Os índices de cobertura vacinal foram muito baixos e inferiores à meta do Ministério da Saúde ${ }^{35} \mathrm{e}$ à meta global do $\mathrm{UNICEF}^{21}$ para 1995, de vacinar pelo menos $80 \%$ das crianças menores de um ano, exceto em relação à BCG. As coberturas vacinais mostraram-se mais baixas do que as dos Estados Unidos, $1995^{8}$, Londrina, $1990^{7}$, Pelotas, $1993^{12} \mathrm{e}$ Brasil, 19964. Foram mais ou menos semelhantes aos níveis observados no Nordeste do Brasil, em $1991^{41}$ e em países asiáticos pobres, como Bangla$\operatorname{desh}^{37}$. As coberturas vacinais foram superiores às 
da Bósnia, em 1993, uma região em guerra civil ${ }^{38}$, e do Maranhão, $1991^{17}$.

A cobertura vacinal considerando-se apenas as crianças de 12 a 23 meses tende a ser mais próxima da cobertura real na população ${ }^{41}$, porém produziu estimativas por intervalo de confiança menos precisas do que as calculadas a partir das crianças de 12 a 59 meses, devido ao menor tamanho amostral neste segmento. As coberturas vacinais, quando se considerou também a informação verbal da mãe ou responsável, foram mais altas do que as obtidas levando-se em conta apenas o registro no cartão, mas este estimador pode estar incluindo também vacinas não recebidas pelas crianças, devido à sua menor confiabilidade.

A associação entre idade de 3 e 4 anos e menores níveis de cobertura vacinal, que permaneceu mesmo após o ajuste para fatores de confusão, está, provavelmente, indicando que as coberturas vacinais estão em ascensão no Município, pois a coorte mais jovem foi mais vacinada que a mais velha. Fala a favor deste argumento também o fato de que a cobertura vacinal em crianças de 12 a 23 meses foi maior do que entre as crianças de 12 a 59 meses. Ao contrário, comparação das estimativas com estudo anterior indica que não houve elevação da cobertura vacinal nos três últimos anos. Entretanto, nesta comparação pode ter ocorrido uma falha em detectar uma diferença estatisticamente significante porventura existente, devido ao baixo poder do teste, pois em 1991 foram estudadas apenas 192 crianças menores de 5 anos e um número ainda mais baixo de crianças de 12 a 23 meses $^{17}$.

Contrariamente a outros trabalhos, que descreveram níveis mais baixos de vacinação dentre os mais pobres ${ }^{3,23,25,37}$, não se observou diferença na cobertura vacinal segundo a renda familiar, demonstrando a necessidade de se desenvolverem esforços para incrementar as coberturas vacinais em todas as faixas de renda. Também em Londrina, não houve diferença na cobertura segundo renda familiar ${ }^{7}$.

Houve diferença na cobertura vacinal segundo o número de irmãos, da mesma forma que o encontrado em outros estudos ${ }^{4,14,25,40,43}$. Entretanto, esta associação desapareceu após o controle dos fatores de confusão, indicando que o maior número de irmãos não foi um fator independentemente associado à nãovacinação.
A posse de seguro-saúde não foi um preditor de maiores níveis de cobertura vacinal, ao contrário dos EUA, onde as crianças com convênio têm maiores taxas de vacinação ${ }^{23,24}$.

A baixa escolaridade materna manteve-se associada com a baixa cobertura vacinal, mesmo após o controle para os fatores de confusão, confirmando observações de outros trabalhos ${ }^{3,4,14,37,40,41,43}$. A baixa escolaridade materna é fator associado com vários eventos relacionados à saúde infantil, como por exemplo, a maiores coeficientes de mortalidade infantil ${ }^{11}$, de morbidade e pior desenvolvimento físico das crianças, além de ser fator inibidor das consultas preventivas de puericultura.

Cleland e Van Ginneken ${ }^{11}$ fizeram revisão dos estudos a respeito dos mecanismos pelos quais a escolaridade materna influencia a mortalidade infantil. Vantagens econômicas associadas com melhor educação (melhor renda, melhores condições de moradia e saneamento) são responsáveis por aproximadamente metade do efeito redutor da escolaridade na mortalidade infantil. Maior uso de serviços de saúde e melhor cuidado doméstico com a criança foram também importantes fatores mediadores. Variáveis reprodutivas (número de filhos, gênero, espaçamento intergestacional e idade materna) são fatores de papel controverso.

O aumento do nível de escolaridade dos pais, especialmente da mãe, propicia maior conhecimento dos problemas de saúde de seus filhos. Há tendência à diminuição de crenças em superstições e explicações mágicas para as doenças. Mães com mais de 8 anos de escolaridade adaptam-se melhor à tecnologia médica e tendem a fazer uso mais efetivo dos serviços de saúde. As mães de maior escolaridade tendem a ter maior acesso, maior conhecimento e possuem maior habilidade para interagir com os serviços de saúde ${ }^{32,34}$. Tendem a valorizar mais o cuidado preventivo, têm maior poder de decisão dentro do núcleo familiar, são menos fatalistas e mais informadas, mais propensas a seguir as orientações médicas, a comprar e usar a medicação prescrita e a adotar novas formas de comportamento, têm maior exposição e compreendem melhor os conteúdos das mensagens educativas ${ }^{11}$.

Vários trabalhos têm mostrado que barreiras estruturais localizadas nos serviços são obstáculos para a vacinação ${ }^{14,29,44,45}$. Estas barreiras não foram estu- 
dadas no presente trabalho mas parece que estão atuando mais sobre crianças de mães de baixa escolaridade, sendo necessário aprofundamento de estudos sobre este tema.

A não-associação entre renda familiar e cobertura vacinal fala a favor de acesso mais igualitário à vacinação, onde os fatores facilitadores (renda e posse de seguro-saúde) não interferiram na vacinação. As barreiras à vacinação se situaram nos fatores predisponentes, especialmente na escolaridade materna ${ }^{1}$. Foi o grupo de mães que freqüentou até 4 anos de escola, cujos filhos apresentaram os níveis mais baixos de cobertura vacinal, sendo este o grupo-alvo para as ações de vacinação. Entretanto, as coberturas vacinais foram baixas para

\section{REFERÊNCIAS}

1. Andersen R, Newman JF. Societal and individual determinants of medical care utilization in the United States. Milkbank Mem Fund Q 1973; 5:95-124.

2. Barroos FC, Victora CG. Epidemiologia da saúde infantil. São Paulo: Hucitec/Unicef; 1991.

3. Becker RA, Lechtig A. Vacinação. In: Silva, RMR, coordenador. Perfil estatístico de crianças e mães no Brasil: situação de saúde 1981. Rio de Janeiro: IBGE; 1984.

4. BEMFAM. Pesquisa nacional sobre demografia e saúde 1996. Rio de Janeiro: BEMFAM/DHS; 1997.

5. Bettiol H, Barbieri MA, Gomes UA, Andrea M, Goldani MZ, Ribeira ERO. Saúde perinatal: metodologia e características da população estudada. Rev Saúde Pública 1998; 32:18-28.

6. Breslow NE. Covariance analysis of censored survival data. Biometrics 1974; 30:89-99.

7. Campos JJB. Estudo das condições de saúde das crianças do Município de Londrina. Ribeirão Preto; 1992. [Dissertação Mestrado - Faculdade de Medicina de Ribeirão Preto da USP].

8. Center for Disease Control. Vaccination coverage levels among children aged 19-35 months - United States, AprilJune 1994. MMWR 1995; 44:396-8.

9. César JA, Victora CG. Avaliando a saúde infantil em uma pequena comunidade: o estudo de Itapirapuã, Vale do Ribeira, SP. Cad Saúde Pública 1990; 6:455-67.

10. Chahnazarian A, Ewbank DC, Makani B, Ekouevi K. Impact of selective primary care on childhood mortality in a rural health zone of Zaire. Int J Epidemiol 1993; 22 (Suppl. 1): S32-S41. todas as faixas de renda e escolaridade, indicando que as ações de vacinação, apesar de amplamente disponíveis no município, não estão conseguindo oferecer alta cobertura. É provável que muitas mães não estejam plenamente convencidas da importância da imunização. Uma das estratégias para o aumento da cobertura vacinal é o incremento das atividades de educação em saúde, no intuito de difundir informação aos grupos-alvo, pois um dos obstáculos à elevação dos níveis de vacinação em São Luís, está, provavelmente, nos conhecimentos e crenças dos usuários. Estudos qualitativos são prioritários para confirmar os achados acima sugeridos e, sendo este o caso, para identificar conteúdos prioritários para discussão de Informação, Educação e Comunicação.

11. Cleland JG, Van Ginneken JK. Maternal education and child survival in developing countries: the search for pathways of influence. Soc Sci Med 1988; 27:1357-68.

12. Costa JSD, Victora CG, Barros FC, Halpern R, Horta BL, Manzolli P. Assistência médica materno-infantil em duas coortes de base populacional no Sul do Brasil: tendências e diferenciais. Cad Saúde Pública 1996; 12 (Suppl. 1): 59-66.

13. Cox DR. Regression models and life tables. J Roy Stat Soc B 1972; 34:187-220.

14. Cutts F, Soares A, Jecque AV, Cliff J, Kortbeek S, Colombo S. The use of evaluation to improve the Expanded Programme on Immunization in Mozambique. Bull World Health Organ 1990; 68:199-208.

15. Dean AG, Dean JA, Coulombier D, Brendel KA, Smith DC, Burton AH, Dicker RC, Sullivan K, Fagan RF, Arner TG. Epi Info: a word processing, database, and statistics program for epidemiology on micro-computers [disquete] Centers of Disease Control and Prevention. Version 6. Atlanta (GA); 1994.

16. Desgrées Du Loû A, Pison G, Aaby P. Role of immunizations in the recent decline in childhood mortality and the changes in the female/male mortality ratio in rural Senegal. Amer J Epidemiol 1995; 142:643-52.

17. Estado do Maranhão/UNICEF. Crianças e adolescentes no Maranhão: saúde, educação e trabalho. São Luís: Governo do Estado do Maranhão/Unicef; 1992.

18. Fundação IBGE. Censo demográfico 1991; Maranhão: resultados do universo relativos às características da população e dos domicílios. Rio de Janeiro; 1991.

19. Fundação IBGE. Contagem da população 1996: dados preliminares; Brasil, Rio de Janeiro; 1997. 
20. Gistelinck F. Carajás: usinas e favelas. São Luís; 1988.

21. Grant J. Situação mundial da infância 1995. Brasília, Unicef; 1995.

22. Henderson RH, Keja J, Hayden G, Galazka A, Clements J, Chan C. Immunizing the children of the world: progress and prospects. Bull World Health Organ 1988; 66:535-43.

23. Himmelstein DU, Woolhandler S. Care denied: US residents who are unable to obtain needed medical services. Am J Public Health 1995; 85: 341-4.

24. Holl JL, Szilagyi PG, Rodewald LE, Byrd RS, Weitzman ML. Profile of uninsured children in the United States. Arch Pediatr Adolesc Med 1995; 149:398-406.

25. Huezo CM, Monteith RS, Naar H, Morris L. Utilización de los servicios de salud de la madre y el niño y cobertura de la inmunización en Panamá. Bol Of Sanit Panam 1982; 93:191-206.

26. Kalton G. Introduction to survey sampling. Beverly Hills: Sage Publications; 1983.

27. Kish L. Survey sampling. New York: John Wiley \& Sons; 1965

28. Kleinbaum DG. Survival analysis: a self-learning text. New York: Springer-Verlag; 1996.

29. Lannon C, Brack V, Stuart J, Caplow M, McNeill A, Bordley C, Margolis P. What mothers say about why poor children fall behind on immunizations - a summary of focus groups in North Carolina. Arch Pediatr Adolesc Med 1995; 149: 1070-5.

30. Lee J. Odds ratio or relative risk for cross-sectional data? Int J Epidemiol 1994; 23:201-3.

31. Levy PS, Lemeshow S. Sampling of populations: methods and applications. New York: John Wiley \& Sons; 1991.

32. McKinlay JB. Some approaches and problems in the study of the use of services - an overview. J Health Soc Behav $1972 ; 13: 115-52$.

33. Menezes AMB, Barros FC, Victoria CG. Tomasi E, Halpern R, Oliveira ALB. Fatores de risco para mortalidade perinatal em Pelotas, RS, 1993. Rev Saúde Pública 1998; 32:209-16.
34. Medina EL, Kaempffer AM, Cumsille FG, Medina RK. Factores de importancia en la demanda de atención médica infantil. Rev Chil Pediatr 1985; $56: 113-9$

35. Ministério da Saúde. Programa Nacional de Imunizações 20 anos. Brasília; 1993.

36. Olsen J, Frische G. Social differences in reproductive health. Scand J Soc Med 1993; 21:90-7.

37. Rahman MM, Islam MA, Mahalanabis D. Mother's knowledge about vaccine preventable diseases and immunization coverage of a population with high rate of illiteracy. J Trop Pediatr 1995; 41:376-81.

38. Robertson A, Fronczak N, Jaganjac N, Hailey P, Copeland P, Duprat M. Nutrition and immunization survey of bosnian women and children during 1993. Int J Epidemiol 1995; 24:1163-70.

39. Silva LMC, Mota ELA. Estudo por inquérito domiciliar da cobertura vacinal no Estado da Bahia. Rev Baiana Saúde Pública 1990; 17:45-79.

40. Szwarcwald CL, Valente JG. Avaliação da cobertura de vacinação em Teresina - Piauí (Brasil - 1983). Cad Saúde Pública 1985; 1:41-9.

41. UNICEF. Saúde e nutrição das crianças nordestinas: pesquisas estaduais 1987-1992. Brasília; 1995.

42. UNICEF/Fundação IBGE. Censo demográfico 1991: indicadores sociais - crianças e adolescentes. Brasília; 1994.

43. Valente JG. Cobertura de vacinação no Rio de Janeiro em 1984. Rio de Janeiro, 1987. [Dissertação de Mestrado Escola Nacional de Saúde Pública da Fiocruz].

44. Weese CB, Krauss MR. A 'Barrier-free' health care system does not ensure adequate vaccination of 2-year old children. Arch Pediatr Adolesc Med 1995; 149:1130-5.

45. Wood D, Pereyra M, Halfon N, Hamlin J, Grabowsky M. Vaccination levels in Los Angeles public health centers: the contribution of missed oportunities to vaccinate and other factors. Am J Public Health 1985; $85: 850-3$. 